\title{
A Repetitive Sampling-based Control Chart for Multivariate Weighted Poisson Distribution with Two Different Indices
}

\author{
Shohreh Enami and Hamazeh Torabi* \\ Yazd University \\ Received: 11/9/2020 Approved: 1/13/2021
}

\begin{abstract}
Control charts using repetitive group sampling have been an attractive topic in the last few years. This paper presents a control chart for multivariate weighed Poisson distribution by repetitive sampling with two different indexes. The effect of these two indexes on the performance of the control chart will be investigated based on the average sequence length criterion. Unlike almost all the research studies on this topic, this paper considers those cases in which the process parameters in the out-of-control situation are not necessarily a constant proportion of the process parameters in the control situation. In this paper, we will show that choosing appropriate statistics can be useful in the performance of the control chart and increasing its efficiency.
\end{abstract}

Keywords. Multi-attribute process control; average run length; multivariate Poisson distribution; repetitive sampling.

MSC 2010: 62D05.

\footnotetext{
${ }^{*}$ Corresponding author
} 


\section{Introduction}

Control charts are important tools in statistical quality control for monitoring and improvement purposes. Control charts are dividable into attributes and variable control charts, depending on whether the quality characteristic is attributable or measurable. When process quality characteristics are classified on the nominal or ordinal scale, the corresponding monitoring process is regarded as a multivariate attribute process control $\mathrm{Lu}$ et al. (1998). It is noteworthy to mention that little work has been carried out on statistical procedures to monitor multivariate attribute processes and many problems in developing multi-attribute monitoring methods are still open Niaki and Akbari-Nasaji (2011); Niaki and Khedmati (2013). To study some reviews on the multi-attribute statistical process, control the readers may refer to Bersimis et al. (2007); Mason and Young (2002); Saghir and Lin (2015); Topalidou and Psarakis (2009). Many research studies focused on control charts for multivariate Poisson distribution. For more discussion, one may refer to Ali Raza and Aslam (2018); Aparisi et al. (2014); Epprecht et al. (2013); Garcia-Bustos et al. (2016); He et al. (2014); Ho and Costa (2009); Holgate (1964); Kuo and Chiu (2008); Laungrungrong et al. (2014); Niaki and Khedmati (2013). Ahmad et al. (2013) and Aslam et al. (2014) offered repetitive sampling in the field of control charts. Recently, Aslam et al. (2017) introduced a control chart for MP count data under repetitive sampling (RS), and showed this chart is more effective in identifying the process shift. Also, Cozzuzoli et al. (2018) proposed a control chart for multivariate weighted Poisson distribution, with a new index. In the present paper, a control chart for multivariate weighted Poisson distribution is proposed with two different indexes, in Section 2. while section 3 depicts a performance comparison of the proposed control charts in terms of ARL. The average run length $(\mathrm{ARL})$ is used to measure the efficiency of a control chart for the detection of the out-of-control manufacturing process Montgomery (2013). Finally, some conclusions are brought in Section 4. Please note that unlike almost all the research studies on this topic, this paper has considered those cases in which the process parameters in the out-of-control situation are not necessarily a constant proportion of the process parameters in the control situation. 


\section{A Control Chart for Weighted Multivariate Pois- son Distribution Using Repetitive Sampling}

For evaluating the production process, a sampling inspection unit is collected from the process. Each identified defect have to be classified in one of the $P$ category. Let $D=\left(D_{1}, \cdots, D_{j}, \cdots, D_{P}\right)$ be a vector of the $p$ defect categories. The component $D_{j}$ indicates the $j$-th defect category; $D_{1}$ is the least serious defect category, and $D_{P}$ is the most severe defect category.

Let $X_{j}, j=1,2, \cdots, P$ be a number of non-conformities or flaws of the quality characteristics which follows jointly $P$-variate Poisson distribution with mean $\lambda_{j}, j=1,2, \cdots, p$. Similar to Karlis (2003); Krishnamoorthy (1951), we use the notation $X \sim M V P_{P}(\Lambda)$, where $\Lambda=\left(\lambda_{1}, \cdots, \lambda_{P}\right)$. Also, we assume that $\operatorname{Cov}\left(X_{i}, X_{j}\right)=0$ for $i \neq j$.

Let $d=\left(d_{1}, \cdots, d_{j}, \cdots, d_{p}\right)$ be a vector of increasing weights associated to the vector $D$ vector, i.e ., $d_{j}<d_{j+1}, j=1,2, \cdots, P-1$. In general, $d_{j}$ indicates the degree of quality loss that the $j$-th defect class introduces into the system. Jones et al. (1999) defined a index as a linear weighted combination of several Poisson random variables. In the other words, for the fixed vectors $d$ and $\Lambda$, the index is

$$
\xi=\sum_{j=1}^{P} d_{j} \lambda_{j} .
$$

The following sampling statistics can be used to monitor the overall defectiveness parameter $\xi$

$$
\hat{\xi}=\sum_{j=1}^{P} d_{j} \hat{\lambda}_{j}=\sum_{j=1}^{P} d_{j} X_{j},
$$

where $X_{j}$ and $\hat{\xi}$ represent the unbiased estimator of the parameter $\lambda_{j}$ and of the index $\xi$, respectively. Cozzucoli and Marozzi (2017) defined a new index of the overall defectiveness based on a modification of (1) and a twosided Shewhart-type multivariate control chart with asymptotic probabilistic limits to monitor the defectiveness or demerit of the process.

Let $\Lambda_{0}=\left(\lambda_{10}, \cdots, \lambda_{p 0}\right)$ be the parameter vector when process is in con- 
trol. Cozzucoli and Marozzi (2017) introduced a statistic $\delta$ as follows

$$
\delta=\sum_{j=1}^{p} d_{j} p_{j},
$$

where $p_{j}=\frac{\lambda_{j}}{\lambda_{0}}$ is a measure of the relative weight of the $j$-th defect class, and $\lambda_{0}=\sum_{j=1}^{p} \lambda_{j 0}$. They used the geometric progressive sequence for the weights, which is $d_{j}=r d_{j-1}$, where $\mathrm{r}$ is the common ratio of the geometric progression. The following sampling statistic may be used

$$
\hat{\delta}=\sum_{j=1}^{p} d_{j} \hat{p}_{j},
$$

where $\hat{p}_{j}=\frac{X_{j}}{\sum_{j=1}^{p} X_{j}}$.

\section{Case 1: proposed index by Jones}

We use (1) as sampling statistics

$$
\hat{\xi}=\sum_{j=1}^{P} d_{j} X_{j},
$$

Where $d_{j}$ and $X_{j}$ has been defined in above. The outer control limits for the proposed control chart are given by

$$
\begin{aligned}
L C L_{1} & =\mu_{\hat{\xi}}-k_{1} \sigma_{\hat{\xi}}=\sum_{j=1}^{p} d_{j} \lambda_{j 0}-k_{1}\left(\sum_{j=1}^{p} d_{j}^{2} \lambda_{j 0}\right)^{\frac{1}{2}}, \\
U C L_{1} & =\mu_{\hat{\xi}}+k_{1} \sigma_{\hat{\xi}}=\sum_{j=1}^{p} d_{j} \lambda_{j 0}+k_{1}\left(\sum_{j=1}^{p} d_{j}^{2} \lambda_{j 0}\right)^{\frac{1}{2}} .
\end{aligned}
$$

Also, the inner control limits for the proposed control chart are designed by

$$
\begin{aligned}
L C L_{2} & =\mu_{\hat{\xi}}-k_{2} \sigma_{\hat{\xi}}=\sum_{j=1}^{p} d_{j} \lambda_{j 0}-k_{2}\left(\sum_{j=1}^{p} d_{j}^{2} \lambda_{j 0}\right)^{\frac{1}{2}}, \\
U C L_{2} & =\mu_{\hat{\xi}}+k_{2} \sigma_{\hat{\xi}}=\sum_{j=1}^{p} d_{j} \lambda_{j 0}+k_{2}\left(\sum_{j=1}^{p} d_{j}^{2} \lambda_{j 0}\right)^{\frac{1}{2}} .
\end{aligned}
$$


- Inner control limits

$L C L_{2}=\max \left[0, \sum_{j=1}^{p} d_{j} \lambda_{j 0}-k_{2}\left(\sum_{j=1}^{p} d_{j}^{2} \lambda_{j 0}\right)^{\frac{1}{2}}\right], \quad U C L_{2}=\sum_{j=1}^{p} d_{j} \lambda_{j 0}+k_{2}\left(\sum_{j=1}^{p} d_{j}^{2} \lambda_{j 0}\right)^{\frac{1}{2}}$.

where $k_{1}$ and $k_{2}$ are the control limit coefficients and selected in such a way that a specific in-control ARL is attained.

Under normal approximation, The out-of-control probability $\left(P_{\text {out.1 }}^{0}\right)$ based on a single sample when the process is in-control is given by

$P_{\text {out.1 }}^{0}=P\left(\hat{\xi} \leq L C L_{1} \mid \lambda_{j}=\lambda_{j 0}\right)+P\left(\hat{\xi} \geq U C L_{1} \mid \lambda_{j}=\lambda_{j 0}\right)=P_{\text {out.1 }}^{0}=2\left[1-\Phi\left(k_{1}\right)\right]$,

where $\Phi($.$) denotes the cumulative distribution function of the standard nor-$ mal distribution. The probability of repetition $\left(P_{r e p}^{0}\right)$ for the introduced control chart is given as the below

$$
\begin{aligned}
P_{r e p}^{0} & =P\left(U C L_{2} \leq \hat{\xi} \leq U C L_{1} \mid \lambda_{j}=\lambda_{j 0}\right)+P\left(L C L_{1} \leq \hat{\xi} \leq L C L_{2} \mid \lambda_{j}=\lambda_{j 0}\right) \\
& =P_{r e p}^{0}=2\left[\Phi\left(k_{1}\right)-\Phi\left(k_{2}\right)\right] .
\end{aligned}
$$

Therefore, the probability of the process being declared to be out-of-control when the process is in control is given as

$$
P_{\text {out }}^{0}=\frac{P_{\text {out }, 1}^{0}}{1-P_{\text {rep }}^{0}}=\frac{2\left[1-\Phi\left(k_{1}\right)\right]}{1-2\left[\Phi\left(K_{1}\right)-\Phi\left(K_{2}\right)\right]}, \quad A R L_{0}=\frac{1}{P_{\text {out }}^{0}} .
$$

Suppose now that the process parameter $\lambda_{j}=\lambda_{j 0}$ is shifted to $\lambda_{j}=\lambda_{j 1}$. Then, the probability of the process being declared to be out-of-control based on the signal sample when the process is shifted is given by

$$
P_{\text {out }, 1}^{1}=P\left[\hat{\xi} \leq L C L_{1} \mid \lambda_{j}=\lambda_{j 1}\right]+P\left[\hat{\xi} \geq U C L_{1} \lambda_{j}=\lambda_{j 1}\right] .
$$

Similarly, the probability of repetition $\left(P_{r e p}^{1}\right)$ when the process is shifted is obtained

$$
P_{r e p}^{1}=P\left[U C L_{2} \leq \hat{\xi} \leq U C L_{1} \mid \lambda_{j}=\lambda_{j 1}\right]+P\left[L C L_{1} \leq \hat{\xi} \leq L C L_{2} \mid \lambda_{j}=\lambda_{j 1}\right] .
$$

So, the probability of process being declared to be out-of-control $\left(P_{\text {out }}^{1}\right)$ when 
the process is shifted is given as follows

$$
P_{\text {out }}^{1}=\frac{P_{\text {out }, 1}^{1}}{1-P_{\text {rep }}^{1}},
$$

The out-of-control ARL $\left(A R L_{1}\right)$ for the shifted process is given as follows

$$
A R L_{1}=\frac{1}{P_{\text {out }}^{1}} .
$$

\section{Case 2: Proposed index by Cozzuzoli}

The outer and inner control limits for the proposed control chart are given by

$$
\begin{aligned}
L C L_{1} & =\mu_{\hat{\delta}}-k_{1} \sigma_{\hat{\delta}}=\sum_{j=1}^{p} d_{j} P_{j 0}-k_{1} \sqrt{\frac{1}{S_{P}}\left[\sum_{j=1}^{p} d_{j}^{2} p_{j 0}-\left(\sum_{j=1}^{p} d_{j} p_{j 0}\right)^{2}\right]}, \\
U C L_{1} & =\mu_{\hat{\delta}}+k_{1} \sigma_{\hat{\delta}}=\sum_{j=1}^{p} d_{j} P_{j 0}+k_{1} \sqrt{\frac{1}{S_{P}}\left[\sum_{j=1}^{p} d_{j}^{2} p_{j 0}-\left(\sum_{j=1}^{p} d_{j} p_{j 0}\right)^{2}\right]}, \\
L C L_{2} & =\max \left[0, \sum_{j=1}^{p} d_{j} P_{j 0}-k_{2} \sqrt{\left.\frac{1}{S_{P}}\left[\sum_{j=1}^{p} d_{j}^{2} p_{j 0}-\left(\sum_{j=1}^{p} d_{j} p_{j 0}\right)^{2}\right]\right]},\right. \\
U C L_{2} & =\mu_{\hat{\delta}}+k_{2} \sigma_{\hat{\delta}}=\sum_{j=1}^{p} d_{j} P_{j 0}+k_{2} \sqrt{\frac{1}{S_{P}}\left[\sum_{j=1}^{p} d_{j}^{2} p_{j 0}-\left(\sum_{j=1}^{p} d_{j} p_{j 0}\right)^{2}\right] .}
\end{aligned}
$$

where $k_{1}$ and $k_{2}$ are the control limit coefficients and selected in such a way that a specific in-control ARL is attained. The probability of the process being declared to be out-of-control when the process is in control is given as

$$
P_{\text {out }}^{0}=\frac{P_{\text {out }, 1}^{0}}{1-P_{r e p}^{0}}=\frac{2\left[1-\Phi\left(k_{1}\right)\right]}{1-2\left[\Phi\left(K_{1}\right)-\Phi\left(K_{2}\right)\right]},
$$

Where $P_{\text {out. } 1}^{0}=P\left(\hat{\delta} \leq L C L_{1} \mid \lambda_{j}=\lambda_{j 0}\right)+P\left(\hat{\delta} \geq U C L_{1} \mid \lambda_{j}=\lambda_{j 0}\right)=2[1-$ $\left.\Phi\left(k_{1}\right)\right]$ and $P_{r e p}^{0}=P\left[U C L_{2} \leq \hat{\delta} \leq U C L_{1} \mid \lambda_{j}=\lambda_{j 0}\right]+P\left[L C L_{1} \leq \hat{\delta} \leq\right.$ $\left.L C L_{2} \mid \lambda_{j}=\lambda_{j 0}\right]=1-2\left[\Phi\left(k_{1}\right)-\Phi\left(k_{2}\right)\right]$. The in-control ARL is defined as

$$
A R L_{0}=\frac{1}{P_{\text {out }}^{0}} .
$$


Suppose now that the process parameter $\lambda_{j}=\lambda_{j 0}$ is shifted to $\lambda_{j}=\lambda_{j 1}$. Then, the probability of the process being declared to be out-of-control based on the signal sample when the process is shifted is

$$
P_{o u t, 1}^{1}=P\left[\hat{\delta} \leq L C L_{1} \mid \lambda_{j}=\lambda_{j 1}\right]+P\left[\hat{\delta} \geq U C L_{1} \mid \lambda_{j}=\lambda_{j 1}\right] .
$$

Similarly, the probability of repetition $\left(P_{r e p}^{1}\right)$ when the process is shifted is obtained

$$
P_{\text {rep }}^{1}=P\left[U C L_{2} \leq \hat{\delta} \leq U C L_{1} \mid \lambda_{j}=\lambda_{j 1}\right]+P\left[L C L_{1} \leq \hat{\delta} \leq L C L_{2} \mid \lambda_{j}=\lambda_{j 1}\right] .
$$

So, the probability of process being declared to be out-of-control $\left(P_{o u t}^{1}\right)$ when the process is shifted is given as follows

$$
P_{\text {out }}^{1}=\frac{P_{o u t, 1}^{1}}{1-P_{r e p}^{1}} .
$$

The out-of-control ARL $\left(A R L_{1}\right)$ for the shifted process is given as follows

$$
A R L_{1}=\frac{1}{P_{\text {out }}^{1}} .
$$

\section{Performance Comparison}

This section compares the performance of the proposed control charts with two different indexes using the ARL. The research uses the data in Table 1, which are exactly represented in Table 1 Cozzucoli and Marozzi (2017), while considering more inspection units. It is assumed that the process deteriorates progressively. Table 1 illustrates the values of ARLs for the proposed control chart with two different indexes for the identical values of specified parameters. As depicted in Table 1, the RS scheme with a defined index by Cozzucoli has significantly smaller ARL values in comparison with the RS scheme with an index defined by Jones.

\section{Discussion and Conclusions}

This paper proposes a control chart for multivariate weighted multivariate Poisson distribution with two different indexes, using repetitive sampling. The efficiency of the proposed chart is compared by out-of-control ARL using 
Table 1. Comparison of ARLs proposed control chart with two different indexes

\begin{tabular}{ccc}
\hline Deterioration & $\begin{array}{c}\text { RS scheme } \\
\text { with defined index by Cozzucoli }\end{array}$ & $\begin{array}{c}\text { RS scheme } \\
\text { with defined index by Jones }\end{array}$ \\
& $A R L_{0}=200, K_{1}=3.18, K_{2}=0.376$ & $A R L_{0}=200, K_{1}=3.18, K_{2}=0.376$ \\
\hline 1 & 1.015 & 63.01 \\
2 & 1.002 & 37.14 \\
3 & 1.0005 & 22.309 \\
4 & 1.00002 & 14.88 \\
5 & 111.17 & 474.48 \\
6 & 1.082 & 162.42 \\
7 & 1.35 & 290.31 \\
8 & 1.012 & 56.87 \\
9 & 1.033 & 71.205 \\
10 & 1.0018 & 21.73 \\
11 & 1.0014 & 64.28 \\
12 & 1.03 & 105.04 \\
13 & 1.0019 & 63.02 \\
14 & 1.003 & 31.86 \\
\hline
\end{tabular}

different deteriorations when $A R L_{0}$ remains unchanged for control charts. The findings of this paper indicate that the proposed chart with a defined index by Cozzacoli yields a small ARL value. So, it can be concluded that this chart performs better. This chart has industrial applications for the evaluation of production processes.

\section{References}

Ali Raza, M. and Aslam, M., (2018). Design of Control Charts for Multivariate Poisson Distribution Using Generalized Multiple Dependent State Sampling. Quality Technology \& Quantitative Management, doi: 10.1080/16843703.2018.1497935.

Aparisi, F., Garcia-Bustos, S. and Epprecht, E.K. (2014). Optimum Multiple and Multivariate Poisson Statistical Control Charts. Quality and Reliability Engineering International, 30, 221234 .

Aslam, M., Srinivasa Rao, G., Ahmad, L. and Jun, C.-H. (2017). A Control Chart for Multivariate Poisson Distribution Using Repetitive Sampling. Journal of Applied Statistics, 44, 123-136.

Bersimis, S., Psarakis, S. and Panaretos, J. (2007). Multivariate Statistical Process Control Charts: an Overview. Quality and Reliability Engineering International, 23, 517-543. 
Cozzucoli, P.C. and Marozzi, M. (2017). Monitoring Multivariate Poisson Processes: A Review and Some New Results. Quality Technology \& Quantitative Management, 15, 53-68.

Epprecht, E.K., Aparisi, F. and Garcia-Bustos, S. (2013). Optimal Linear Combination of Poisson Variables for Multivariate Statistical Process Control. Computers 85 Operations Research, 40, 3021-3032.

Garcia-Bustos, S., Mite, M. and Vera, F. (2016). Control Charts with Variable Dimension for Linear Combination of Poisson Variables. Quality and Reliability Engineering International, 32, $1741-1755$

He, S., He, Z. and Wang, G.A. (2014). CUSUM Control Charts for Multivariate Poisson Distribution. Communications in Statistics-Theory and Methods, 43, 1192-1208.

Ho, L.L. and Costa, A.F.B. (2009). Control Charts for Individual Observations of a Bivariate Poisson Process. The International Journal of Advanced Manufacturing Technology, 43, 744755.

Holgate, P. (1964). Estimation for the Bivariate Poisson Distribution. Biometrika, 51, 241287.

Jones, L. A., Woodall, W.H. and Conerly M.D. (1999). Exact Properties of Demerit Control Chart. Journal of Quality Technology, 31, 207-215.

Karlis, D. (2003). An EM Algorithm for Multivariate Poisson Distribution and Related Models. Journal of Applied Statistics, 30, 63-77.

Krishnamoorthy, A.S. (1951). Multivariate Binomial and Poisson Distribution, Sankhya, 11, 117-124.

Kuo, T. and Chiu, J. (2008). Regression-based Limits for Multivariate Poisson Control Chart. Paper presented at the Industrial Engineering and Engineering Management, 2008. IEEM 2008. IEEE International Conference on.

Laungrungrong, B., Borror, C.M. and Montgomery, D.C. (2014). A One-sided MEWMA Control Chart for Poisson Distributed Data. International Journal of Data Analysis Techniques and Strategies, 6, 15-42.

Lu, X.S., Xie, M. and Goh T.N. (1998). Control Chart for Multivariate Attribute Process. International Journal of Production Research, 36, 3477-3489.

Mason, R.L. and Young, J.C. (2002).Multivariate Statistical Process Control with Industrial Applications. The American Statistical Association and the Society for Industrial and Applied Mathematics, ASA-SIAM, Philadelphia.

Montgomery, D.C. (2013). Design and Analysis of Experiments. Inc, Cary, NC, JohnWiley \& Sons. 
Niaki, S.T.A. and Akbari-Nasaji, S. (2011). A Hybrid Method of Artificial Neural Network and Simulated Annealing in Monitoring Autocorrelated Multi-attribute Processes. The International Journal of Advanced Manufacturing Technology, 56, 777-788.

Niaki, S.T.A. and Khedmati, M. (2013). Estimating the Change Point of the Parameter Vector of Multivariate Poisson Processes Monitored by a Multi-attribute T 2 Control Chart. The International Journal of Advanced Manufacturing Technology, 1-18.

Niaki, S.T.A. and Khedmati, M. (2013). Estimating the Change Point of the Parameter Vector of Multivariate Poisson Processes Monitored by a Multiattribute T2 Control Chart. The International Journal of Advanced Manufacturing Technology, 64, 1625-1642.

Saghir, A. and Lin, Z. (2015). Control Charts for Dispersed Count Data: an Overview. Quality and Reliability Engineering International, 31, 725-739.

Topalidou, E. and Psarakis, S. (2009). Review of Multinomial and Multiattribute Quality Control Charts. Quality and Reliability Engineering International, 25, 773-804.

\section{Shohreh Enami}

Department of Statistics,

Yazd University,

Yazd, Iran.

email: enami1387@yahoo.com

\section{Hamzeh Torabi}

Department of Statistics

Yazd University

Yazd, Iran.

email: hamzeh.torabi@gmail.com 